\title{
Before Dinner
}

National Cancer Institute

\section{Source}

National Cancer Institute. Before Dinner. NCI Thesaurus. Code C92682.

To be done prior to eating dinner. 